\title{
EFFICIENT AND INNOVATIVE POLISHING PROCESSES FOR SMOOTHING COMPLEX SURFACE GEOMETRIES AND INTERNAL CONTOURS ON BRITTLE- HARD COMPONENTS
}

\author{
Marcel Binder ${ }^{*}$, Sebastian Henkel ${ }^{1}$, Anne-Marie Schwager ${ }^{1}$, Christoph Letsch ${ }^{1}$, Jens Bliedtner ${ }^{1}$, \\ , Vladislav Matusevich² and Andrew Matusevich ${ }^{2}$ \\ ${ }^{1}$ Ernst-Abbe University of Applied Sciences Jena, Carl-Zeiss-Promenade 2, 07745 Jena, Germany \\ ${ }^{2}$ VM-TIM GmbH, Hans-Knöll-Str. 6, 07745 Jena, Germany
}

\begin{abstract}
The material fused silica, as well as other brittle-hard materials such as glass ceramics, have great potential for use in a wide range of applications due to their special material properties. The technical advantages of these materials require sophisticated processing technologies, including polishing steps, in order to be able to use these interesting materials advantageously. In addition, a current trend in modern optical manufacturing is the use of free-form surfaces and monolithic components that combine several optical and mechanical functions in one part. Novel or improved processes are needed in order to meet future requirements for resource-saving and effective production methods at the same time.
\end{abstract}

\section{Methods - A Novel Process Chain}

The production of complex glass components involves a great deal of effort and requires the use of advanced $\mathrm{CNC}$ technology. Especially the final surface treatment to create transparent, optically usable surfaces is a timeconsuming and costly task. Because of this a new process chain was developed which is described below

Ultrasonic-assisted grinding is applied as a first step, to produce workpieces of any shape as well as free-form surfaces. The special kinematic principle, which is accompanied by high-frequency longitudinal tool oscillation, enables efficient manufacturing processes. By the determined reduction of process forces using ultrasound, either higher removal rates or a more gentle processing for tool and material can be achieved. [1] The surfaces produced in this way enable the application of new types of smoothing processes, which have considerable advantages over conventional polishing. The application of modern ultra-fine grinding processes with novel resin-bond tools enables average surface roughnesses which are already comparable to conventional pre-polishing. These tools offer numerous advantages in the CNC-controlled glass processing compared to conventional diamond tools. The diamond grains (D16) are embedded in a special resin bond, which has a certain elasticity comparable to polishing pads. This results in a sort of ductile grinding mode and a lower resulting roughness with already semi-transparent glass surfaces. The roughness values can be further reduced in a very short time by subsequent polishing with $\mathrm{CO}_{2}$ laser. With this process, very short polishing times of
$4.8 \mathrm{~s} / \mathrm{cm}^{2}$ are possible, while at the same time a high surface quality can be achieved. [2] Polishing with $\mathrm{CO}_{2}$ laser radiation is a thermal process in which the energy of the laser radiation is converted into thermal energy. Due to the resulting material heating and reduction of the viscosity at the sample surface, the material starts to flow. The surface roughness is smoothed by the surface tension, roughness peaks are removed and roughness grooves are filled in. [2] Another polishing process that is considered is the wet-chemical polishing of the described optical components. In contrast to laser beam polishing, there is no smoothing of the surface, but a removal of the upper defective material layer. This is achieved by etching the glass in liquid solutions using hydrofluoric acid (HF) and sulphuric acid (H2SO4). [3] As a special feature, ultrasonic support will be used for the first time in the planned experiments to increase process efficiency.

The presented development of a novel process chain consisting of ultrasonic grinding, ultra-fine grinding and the polishing processes laser beam polishing and wetchemical polishing thus offers the possibility of a flexible and fast production of complex glass components, while still ensuring a high optical quality of the surfaces produced.

In addition to the complex outer contour requirements, there are also geometrical requirements for inner contours of complex glass elements, that require a very high aspect ratio (e.g. component length to component diameter). The special challenge here is the high quality of the drill hole wall, which must correspond to a nearly polished surface. The methods of ultrasonic-assisted grinding, ultra-fine grinding, laser beam polishing (LBP) and wet-chemical

\footnotetext{
* Corresponding author: Marcel.Binder@eah-jena.de
} 
polishing described above should also be applied here. Figure 1 shows a schematic representation of the desired process chain.

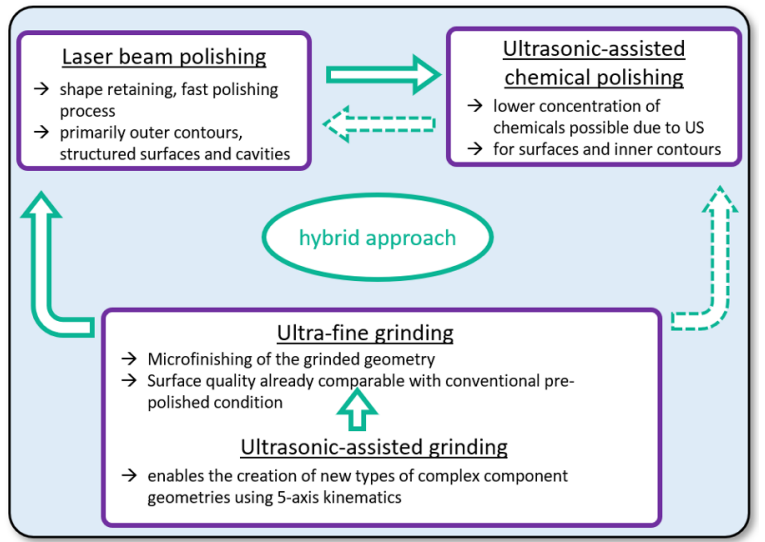

Fig. 1. schematic representation of the novel process chain

\section{Examinations \& Results}

First results of the novel process chain are presented below. The methods were initially applied to external contours in order to gain a basic understanding of the process and to investigate the influence of LBP. Subsequently, first inner contours were realized on which the described polishing processes are soon to be tested.

The novel process chain was investigated using the example of a free-form made of fused silica. Grinding and ultra-fine grinding were performed with a spherical tool (ø $20 \mathrm{~mm}$ ). A meander pattern was chosen with a line distance of $0.1 \mathrm{~mm}$ and a quasi point tool contact. This kinematic approach resulted in narrow grooves, which are visible in the direction of grinding. These can be smoothed by laser beam polishing carried out afterwards. Low roughness values of max. $R_{q}=72 \mathrm{~nm}$ can be already achieved after ultra-fine grinding. With the help of LBP and smoothing of the resulting grooves, the roughness can be reduced even further, to $\max . R_{q}=12 \mathrm{~nm}$. All roughness values were obtained by tactile measurement.

In order to investigate the feasibility of introducing inner contours with a large aspect ratio, drill holes with different diameters $(2 \mathrm{~mm}, 3 \mathrm{~mm}, 5 \mathrm{~mm}, 7.5 \mathrm{~mm} \& 10$ $\mathrm{mm}$ ) and a length of $50 \mathrm{~mm}$ were drilled into samples made of fused silica (Corning HPFS 79805F), ZERODUR $^{\circledR}$ and CLEARCERAM ${ }^{\circledR}$. In addition, diamond drills of different diameters $(2 \mathrm{~mm}, 5 \mathrm{~mm} \& 10$ $\mathrm{mm}$ ) were used for this purpose, whereby the matching bore diameters were realized by using a deep drilling process. The remaining diameters $(3 \mathrm{~mm} \& 7.5 \mathrm{~mm})$ were drilled by using helical machining kinematics. Of particular interest were the resulting roughnesses of the borehole walls after the grinding / drilling process, which are compared in figure 2 . The measurements were taken at the tool entry point as well as at the tool exit point to determine any roughness differences in the course of the hole.

Although the cutting speeds used were calculated to be approximately constant for each bore diameter, it is apparent that there are differences in roughness depending on the material, bore diameter and measuring point.

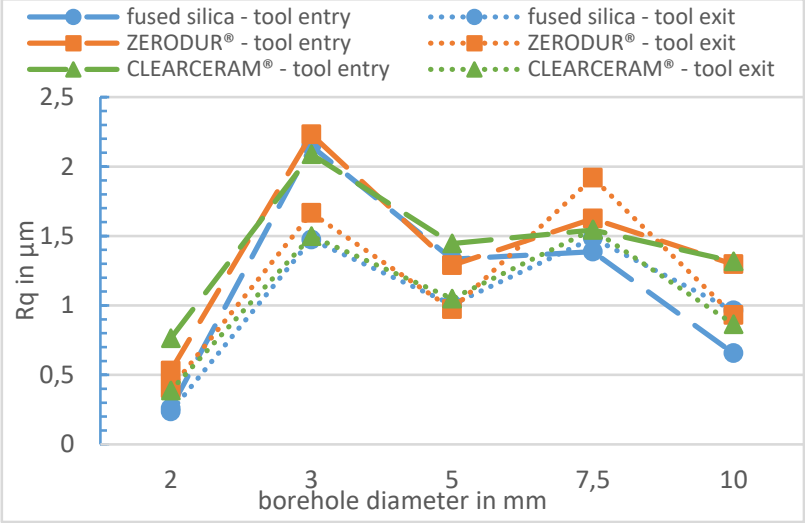

Fig. 2. measured roughness $R q$ at the tool entry and exit points

It can be seen, that for all materials, the $2,3 \& 5 \mathrm{~mm}$ diameter holes have higher roughness at the tool entry than at the tool exit. The same applies to the $10 \mathrm{~mm}$ diameter, with the exception of fused silica. Possibly the material has an influence on the process result here, which is currently still being investigated. In addition, the roughness at the tool entry tends to be lower or the same for all three materials for the $7.5 \mathrm{~mm}$ diameter borehole. The roughness differences between the two machining techniques are most noticeable. The roughness values for helical machining are generally higher ( $3 \mathrm{~mm} \& 7.5 \mathrm{~mm}$ ). The optimal parameter combinations for both kinematics are also currently being investigated.

\section{Conclusion and Outlook}

It could be demonstrated that the new process chain enables a fast and drastic reduction of roughness on complex outer geometries of brittle-hard materials. In the future it will be necessary to investigate whether this process chain can be used for the machining of internal contours with a high aspect ratio. The technical implementation of ultrafine grinding and laser beam polishing will be tested on the bores shown. Furthermore, the wet-chemical polishing for external and internal contours is to be investigated and compared with the described process chain in terms of economic efficiency and achievable surface qualities.

The authors gratefully acknowledge financial support by the German Federal Ministry of Economy and Energy in the funding program ZIM (funding reference: ZF4039613TV8).

\section{References}

1. M. Rost, J. Bliedtner, S. Henkel: Einflüsse des ultraschallunterstützten Schleifens auf silikatische Werkstoffe. In: Eltmann: Diamond Business 1/2016, pp. 68-77. (2016)

2. S. Henkel, A.-M. Schwager, J. Bliedtner, et al.: New surface smoothing technologies for manufacturing of complex shaped glass components. In: Proceedings of SPIE Vol. 10448. (2017)

3. H. Junjiang, Y. Jungxin, C. Wie, Z. Changhe: Experimental investigation of en-chancing the subsurface damage threshold of Nd-doped phosphate glass, In: Chinese Optics Letters, Vol. 6, Issue 9, pp. 681-684. (2008) 